\title{
Machine learning-based automated yeast cell counting under a complicated background with ilastik and ImageJ
}

\author{
Chenxi $\mathrm{Li}^{1}$, Xiaoyu $\mathrm{Ma}^{1}$, Jing Deng ${ }^{1}$, Jiajia $\mathrm{Li}^{1}$, Yanjie $\mathrm{Liu}^{2}$, Xudong Zhu ${ }^{1}$, Ping Zhang ${ }^{1}$, \\ and Jin $\mathrm{Liu}^{1}$ \\ ${ }^{1}$ Beijing Normal University College of Life Sciences \\ ${ }^{2}$ Affiliation not available
}

March 12, 2021

\begin{abstract}
Measuring the concentration and viability of yeast cells is an important and fundamental procedure in scientific research and industrial fermentation. In consideration of the drawbacks of manual cell counting, large quantities of yeast cells require methods that provide easy, objective, and reproducible high-throughput calculations, especially for samples in complicated backgrounds. To answer this challenge, we explored and developed an easy-to-use yeast cell counting pipeline that combined the machine learning-based ilastik tool with the freeware ImageJ, as well as a conventional photomicroscope. Briefly, learning from labels provided by the user, ilastik performs segmentation and classification automatically in batch processing mode for large numbers of images and thus discriminates yeast cells from complex backgrounds. The files processed through ilastik can be recognized by ImageJ, which can set up customizable parameters based on cell size, perimeter, roundness and so on. In this work, we programmed an ImageJ macro, "Yeast Counter", to compute the numeric results of yeast cells for automatic batch processing. Taking the yeast Cryptococccus deneoformans as an example, we observed that the customizable software algorithm for yeast counting with ilastik and ImageJ reduced inter-operator errors significantly and achieved accurate and objective results in the spotting test, while manual counting with a haemocytometer exhibited some errors between repeats and required more time. In summary, a convenient, rapid, reproducible and extremely low-cost method to count yeast cells is described here that can be applied to multiple kinds of yeasts in genetics, cell biology and industrial fermentation.
\end{abstract}

\section{Biotech Methods}

Machine learning-based automated yeast cell counting under a complicated background with ilastik and Image J

Chenxi $\mathrm{Li}^{1}$, Xiaoyu Ma ${ }^{1}$, Jing Deng ${ }^{1}$, Jiajia $\mathrm{Li}^{1}$, Yanjie Liu ${ }^{1}$, Xudong Zhu ${ }^{1}$, Ping Zhang ${ }^{{ }^{*}}$ and Jin Liu ${ }^{2^{*}}$

1 Beijing Key Laboratory of Genetic Engineering Drug and Biotechnology, Institute of Biochemistry and Molecular Biology, College of Life Sciences, Beijing Normal University (CLS-BNU), Beijing 100875, China

2 Beijing Key Laboratory of Gene Resources and Molecular Development, College of Life Sciences, Beijing Normal University (CLS-BNU), Beijing 100875, China

*Corresponding author

Correspondence Ping Zhang:zp1516@bnu.edu.cn

Correspondence Jin Liu:liujin@bnu.edu.cn

\section{Abstract}


Measuring the concentration and viability of yeast cells is an important and fundamental procedure in scientific research and industrial fermentation. In consideration of the drawbacks of manual cell counting, large quantities of yeast cells require methods that provide easy, objective, and reproducible high-throughput calculations, especially for samples in complicated backgrounds. To answer this challenge, we explored and developed an easy-to-use yeast cell counting pipeline that combined the machine learning-based ilastik tool with the freeware ImageJ, as well as a conventional photomicroscope. Briefly, learning from labels provided by the user, ilastik performs segmentation and classification automatically in batch processing mode for large numbers of images and thus discriminates yeast cells from complex backgrounds. The files processed through ilastik can be recognized by ImageJ, which can set up customizable parameters based on cell size, perimeter, roundness and so on. In this work, we programmed an ImageJ macro, "Yeast Counter", to compute the numeric results of yeast cells for automatic batch processing. Taking the yeastCryptococccus deneoformans as an example, we observed that the customizable software algorithm for yeast counting with ilastik and ImageJ reduced inter-operator errors significantly and achieved accurate and objective results in the spotting test, while manual counting with a haemocytometer exhibited some errors between repeats and required more time. In summary, a convenient, rapid, reproducible and extremely low-cost method to count yeast cells is described here that can be applied to multiple kinds of yeasts in genetics, cell biology and industrial fermentation.

[Key words]Yeast, ImageJ, ilastik, haemocytometer, batch processing

\section{Introduction}

Assessing cell viability is a convenient and fundamental method to analyse the effects of various stressors on yeast cells in scientific research and in any brewing process, in which cell counting-associated technologies, such as concentration calculations and spotting tests, are widely adopted to provide an estimation of viable yeast cells [1]. These assays can be used to measure the results of yeast proliferation; to test the growth rate of yeast cells under different kinds of chemical, physical, or environmental factors; and as an internal control to achieve consistent fermentations in industry. At present, commonly used cell counting methods include plate counting [2], real-time quantitative PCR [3], haemocytometers [4], automatic cell counting instruments [5] and flow cytometry counting in biological operation.

The plate counting method is performed by spreading living cells on solid media to form colony forming units (CFUs), which can be counted with the naked eye. A corresponding method for automatic colony counting with ImageJ software has been developed [6]. The advantage of this method is that non-viable yeast cannot duplicate and form colonies on plates, but some shortcomings of this method are that the number of yeast cells only depends on different dilution concentrations and clumped cells will be registered as one count. Additionally, plate counting is also time consuming. Real-time quantitative PCR involves the application of related instruments and the drawing of accurate standard curves. Of course, this method relies on advanced expensive equipment and requires the pre-establishment of control genes, high-efficiency primers or the fluorescent dye PMA [7]. Most cell counters can only count specific types of cells. Professional yeast counters used in fermentation engineering are always expensive and may be assisted with stains that are unfavourable to the operator. In addition, flow cytometry changes the breakpoint of droplets so that the size of droplets formed by sheath fluid can wrap a cell [8], but it demands a more uniform cell size and can be better detected when cells exhibit certain fluorescence signals. However, the size of yeast cells varies widely, and a droplet may contain multiple yeast cells. As a consequence, it is impossible to count yeast cells accurately without the insertion of a fluorescent protein. Therefore, haemocytometer counting remains the most commonly used technique to determine the concentration of a yeast sample because of its ease of use and low cost $[9 ; 10 ; 11 ; 12 ; 13]$.

Problems do exist with the haemocytometer method, such as it being time consuming and inefficient to implement for large-scale analyses. Using a photomicroscope, some researchers have developed effective software to automatically count cells on haemocytometer plates to avoid subjective manual counting and high-throughput statistics. However, some of these methods are limited to specific types of cells. For example, CellProfiler [14] can count mammalian and non-mammal cells via a high-throughput analysis. However, non- 
mammalian cells are limited to round cells, fission yeasts and breeding budding yeasts. CellC [15]can only count labelled cells. CellCounter [16]and OpenCFU [17] are designed for specialized cells and cannot count high concentrations of yeast. Moreover, in cases in which a non-homogeneous liquid is used to test yeast viability or there is background material in the fermentation process, making yeast cell counting even more challenging. Therefore, we intended to introduce the use of the free ilastik and ImageJ software for batch enumeration of yeast cells in complicated backgrounds.

As an open source image analysis program that can be run under the Macintosh, Windows and Linux operating systems, ImageJ has been reported to be suitable for mammalian cell counting [18]. Considering that mammalian cells with an irregular morphology generally grow and adhere to the wall and that their cell size is usually larger than that of yeast, mammalian cells are much easier to count than yeast cells. Hence, whether ImageJ can be used for yeast cell counting remains to be determined. Moreover, the culture media of mammalian cells has fewer impurities, and the background is easy to separate from cells in automatic counting. However, depending on the different purposes of yeast cultures, impurities in yeast media vary tremendously in type and quantity, which would interfere with the automatic counting of yeast cells by ImageJ. Currently, ilastik software can solve this problem very well. ilastik is easy to operate and can provide end users with machine learning-based image analysis [19] without substantial computational expertise; thus, we mainly use the workflow of ilastik to segment and classify images to maximize the separation of yeast cells from the background.

Taking the yeast Cryptococccus deneoformans as an example, a new rapid automated yeast cell counting method using ilastik and ImageJ was assessed in this study. As an opportunistic pathogenic fungus, the spotting test is frequently used to study the sensitivity of $C$. deneoformans cells to various environmental stresses and drugs, in which cell counting is the first step. Here, we describe an ImageJ macro, named "Yeast Counter", and systematically test its performance for counting yeast cells. For samples in complicated backgrounds, the ilastik workflow was able to perform segmentation and classification with interactively supervised machine learning. Then, the number of yeast cells was counted using the "Yeast Counter" macro, which can set up customizable parameters based on cell size, perimeter, roundness and so on in the batch processing mode. According to the results of the spotting test, we observed that the customizable software algorithm for yeast counting reduced inter-operator errors significantly and generated accurate and objective results, while manual counting with a haemocytometer exhibited some errors between repeats and required more time than "Yeast Counter".

\section{Experimental Section}

\subsection{Yeast strains and culture media}

C. deneoformans var. deneoformans JEC21 (serotype D) was used as the experimental subject. Liquid and solid YPD media (2\% glucose, $2 \%$ peptone, $1 \%$ yeast extract, and $\mathrm{pH} 6.0$ ) were used for routine growth and spotting tests. The yeast was cultured for 18 hours to the exponential phase, and the cells were collected by centrifugation and resuspended in liquid YPD, PBS buffer or sterile water. The yeast samples were diluted to make the number of cells in each smallest square of the haemocytometer average 5 to 25 .

\subsection{Haemocytometer}

The gridded area of the haemocytometer plate consisted of nine $1 \times 1 \mathrm{~mm}\left(1 \mathrm{~mm}^{2}\right)$ squares. These were subdivided into 3 sections: $0.25 \times 0.25 \mathrm{~mm}\left(0.0625 \mathrm{~mm}^{2}\right), 0.25 \times 0.20 \mathrm{~mm}\left(0.05 \mathrm{~mm}^{2}\right)$ and $0.20 \times 0.20 \mathrm{~mm}$ $\left(0.04 \mathrm{~mm}^{2}\right)$. The central square was further subdivided into $0.05 \times 0.05 \mathrm{~mm}\left(0.0025 \mathrm{~mm}^{2}\right)$ squares. The remaining squares were not used. The raised edges of the haemocytometer plate hold the coverslip $0.1 \mathrm{~mm}$ off the marked grid, giving each square a defined volume (https://en.wikipedia.org/wiki/Hemocytometer). Cells in suspensions were deposited into a haemocytometer.

concentration of yeasts in original mixture $=\left(\frac{\text { number of yeasts counted }}{(\text { proportion of }}\right.$

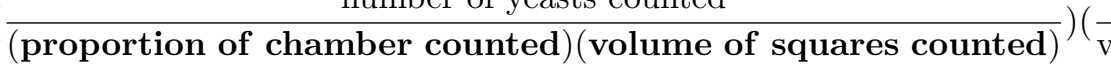




\subsection{Microscope and image capture}

A ZEISS Apotome 2 microscope (Zeiss, Heidenheim, Germany) was used to take pictures of different samples on the haemocytometer with the same parameters (bright field, 20x objective lens, $27.36 \mathrm{msec}$ exposure time). Due to the limited field of view in microscope imagery, each picture included 120 counting chambers. For ease of observation, the images were processed, and the 16 smallest counting chambers are displayed in Figure 1A and Supplementary Figure (Fig.SA), with or without a complex background. As a result, a total of 72 images were collected from the two groups (36 with simple backgrounds and 36 with complex backgrounds).

\subsection{Image compression}

ImageJ was used as the preferred platform for image processing. Users need to select Plugins-Macros-Record commands in ImageJ to record the processing procedure and create a new macro for batch processing. The first step is to convert the RGB image captured by the high-definition microscope into 8 bits and resize the image (see "image compress.ijm" in the supplementary material). All the images were converted automatically to greyscale, and the size was compressed in batches by the Process-Batch-Macro commands according to the recorded processing method, as shown in Figure 1B and Fig.SB.

\subsection{Machine learning-based segmentation and classification}

ilastik was used to distinguish the background from the yeast to be counted. For example, the pixel classification function of the software was used to remove the background of the compressed pictures in batches. The background and yeast samples can be marked many times by different labels under the Training-Live update command in the process of background removal. Regardless of whether the background was simple or complex, it was separated from the yeast to be counted as much as possible (Figure 1C and Fig.SC).

\subsection{Establishment and application of ImageJ macro"Yeast Counter"}

The ImageJ macro "Yeast Counter" was written in a Java-like programming language, and full operational details are provided in the "Yeast Counter" in the supplementary material. We smoothed and adjusted the threshold of the images processed in batches using ilastik and ImageJ (Image-Adjust-Threshold-Li-ProcessSmooth-apply), and subsequently black-and-white images were presented. Then, the Process-Binary-Fill hole and Watershed functions in ImageJ were used to fill the gap (the part marked by the red circle in Figure 1D, Fig.SD) and separate joined cells (the position indicated by the red clipper in Figure 1E, Fig.SE). In addition, Analyse-Analyse Particles - Size, Area, Circ and other indexes can be used to assess the objectives in images. Here, only the Area command was applied to distinguish yeast cells from background fragments (Figure 1F and Fig.SF). Then, the threshold in the Analyse Particles command was set, and the number of yeast cells was counted automatically (Figure 1G and Fig.SG, "count.ijm" in the supplementary material). The final results were saved as a file in csv format.

\subsection{Spotting test}

To compare the effect of manual and automatic counting, a spotting test was applied. Briefly, the concentration of yeast cells in the solution was calculated through the haemocytometer formula according to the results of the manual counting and the "Yeast Counter" macro. Samples with the maximum concentration were taken and diluted to $2 \times 10^{7}$ cells per ml, which were subsequently subjected to 10 -fold dilutions. Five microlitres of each dilution, whose concentration ranged from $2 \times 10^{7}$ to $2 \times 10^{3}$ cells per ml, were spotted onto YPD solid media and incubated at $30{ }^{\circ} \mathrm{C}$ for 3 to 5 days. After incubation, comparisons between the manual and automatic groups were performed, in which extra care was taken for the spots with the fewest cells (Figure 2).

\section{Results}

\subsection{Comparison of effectiveness and timein manual and automatic counting}

The biggest disadvantage of manual yeast cell counting with a haemocytometer is that the process is very time consuming and inefficient in batch processing; thus, the first assessment index of the automatic counting 
procedure is its efficiency. Manual and automatic counting were used simultaneously to analyse the same yeast samples in this study regardless of whether the samples were in simple or complex backgrounds. According to the principle of manual haemocytometer counting, in a complex background, it took an average of at least 1-2 minutes to count cells in one middle square, which was further divided into 16 smaller squares, and the total time for one sample was approximately 5-10 minutes by trained observers. To reduce errors, the counting process was repeated three times, which made the total analysis time for each sample approximately 15-30 minutes, and the total counting time for 12 samples was at least 2 hours. In contrast, when yeast cells were counted automatically, a trained operator took approximately 5-10 minutes to take 36 images (3 images per sample included 400 small squares that needed to be counted), 2-5 minutes to set the parameters, and 1-2 minutes for the automatic PC calculation. Therefore, automatic counting in this study took only 2 to 5 percent of the time required for manual counting, which greatly improved the efficiency of the experiment (Figure 3A). These processing times can be further improved after the first operation for samples in the same backgrounds due to the use of the reproducible "Yeast Counter" macro, which made the average counting time for one sample 2-5 minutes. These results demonstrated the high efficiency of yeast cell counting with ilastik and ImageJ in a complex background.

\subsection{Higher accuracy exhibited by the automatic method}

Subjective factors can play a role in the yeast cell counting process with a haemocytometer; for example, some cells can be half-in/half-out of the square being counted, and it is difficult to be consistent throughout manual counting. Moreover, for budding yeast cells, the standard of sub-cells with different sizes that can be counted as a single individual varies by different observers, and the same observer would also obtain different statistical results. As the software standard can be unified by the operator, we speculated that the automatic yeast cell counting method would be more accurate than manual counting. Thus, the spotting test was applied in this study. Briefly, 5 out of 12 yeast samples in complex backgrounds or not were taken and diluted to the same concentration and then subjected to 10 -fold dilutions. As the number of yeast cells is theoretically consistent at the same dilution, it is obvious that the results of manual counting exhibited a greater deviation between samples (Figure 2A and 2C), while those of automatic counting showed better consistency (Figure 2B and 2D) whether the background was complex or not. We also calculated the CFUs (colony-forming units) of the last spot on YPD media, which were anticipated to be 10 in theory according to the formula for concentration. The results revealed that the average value of the CFUs in automatic counting was 7.8 , while the value for manual counting was 1.8 (Figure 3B), demonstrating the higher accuracy of the automatic method.

\section{Discussion}

Yeasts are unicellular organisms that are widely used in genetics, cell biology and industrial fermentation. The automatic counting method used in this research can be applied to multiple kinds of yeasts, such asSaccharomyces cerevisiae, Schizosaccharomyces pombe, andPichia pastoris . Compared with manual counting and expensive cell counting instruments, the automated method described here is convenient, rapid, reproducible and extremely low cost. An ordinary microscope that can take photographs, as well as the freeware ilastik and ImageJ, are the only tools used in the method, which is easy to manipulate after a short training time, saving both time and reducing human subjective evaluation. In addition, the method can also be widely used in other fungal experiments, such as counting fungal spores when calculating the spore germination rate of filamentous fungi. Organelles and cell structures can also be quantitatively analysed when combined with the use of fluorescent dyes and fluorescent fusion proteins. As a consequence, this method is simple and flexible and has a wide range of applications.

The combination of ilastik and ImageJ in yeast cell counting gives full play to their respective advantages, in which ilastik is used for segmentation and classification based on deep learning and an ImageJ macro performs cell counting by adjusting various parameters. ImageJ also has the ability to distinguish between background and cells (Plugins-Segmentation-Trainable Weka Segmentation). However, in the processing process of ImageJ, the efficiency of dealing with a single image is much lower than that of ilastik. Moreover, Fiji cannot learn multiple images at one time. Thus, ilastik is the optimal choice for segmentation with 
deep learning-based high accuracy and high throughput. For the freeware ilastik, annotations of background (Lable1) and individual objects (Lable2) serve as inputs into a regression random forest that estimates the object density in every pixel of the image [20]. However, the "Cell Density Counting" function in ilastik cannot be directly used to obtain an accurate number of yeast cells. Taking the capsule-surrounded $C$. deneoformans as an example, multiple bright spots in the background and the halo of light generated by the capsule around the yeast cell would be recognized as the same pixel as the cells and thus entered into the analysis of cell numbers. This makes the number of yeast cells counted by ilastik much higher than the actual number.

However, there is no doubt that this method has some problems; for example, different batches of image data may have different background depths and complexities. At the time, it is necessary for ilastik software to distinguish and learn each of the different batches of images as it cannot automatically homogenize the pictures, which can be used as an improvement point in future software development. However, what is certain is that the combination of ImageJ and ilastik can greatly reduce the experimental time and lead to greater accuracy when a large number of yeast samples need to be dealt with in batch processing, especially in complex backgrounds. Future implementations of this method will enable the differentiation between dead and live cells by specific fluorescence.

Acknowledgement:

This work was supported by the National Natural Science Foundation of China (NSFC grants \#31900130) and the Fundamental Research Funds for the Central Universities (grants \#2019NTST12).

[1] M. Kwolek-Mirek, R. Zadrag-Tecza, Comparison of methods used for assessing the viability and vitality of yeast cells. FEMS Yeast Res 14 (2014) 1068-79.

[2] C. Wilson, R. Lukowicz, S. Merchant, H. Valquier-Flynn, J. Caballero, J. Sandoval, M. Okuom, C. Huber, T.D. Brooks, E. Wilson, B. Clement, C.D. Wentworth, A.E. Holmes, Quantitative and Qualitative Assessment Methods for Biofilm Growth: A Mini-review. Res Rev J Eng Technol 6 (2017).

[3] G.M. Knight, E. Dyakova, S. Mookerjee, F. Davies, E.T. Brannigan, J.A. Otter, A.H. Holmes, Fast and expensive (PCR) or cheap and slow (culture) A mathematical modelling study to explore screening for carbapenem resistance in UK hospitals. BMC Med 16 (2018) 141.

[4] B. Song, B. Zhuge, H. Fang, J. Zhuge, [Analysis of the chromosome ploidy of Candida glycerinogenes]. Wei Sheng Wu Xue Bao 51 (2011) 326-31.

[5] J. Tang, F.U. Qiangqiang, M. Chen, L.I. Chunmei, L.U. Jiazheng, G.P. University, Volume change of H9c2 cells treated with norepinephrine. Shandong Medical Journal (2018).

[6] N. Stolze, C. Bader, C. Henning, J. Mastin, A.E. Holmes, A.L. Sutlief, Automated image analysis with ImageJ of yeast colony forming units from cannabis flowers. J Microbiol Methods 164 (2019) 105681.

[7] Z. Huang, J. Zheng, C. Shi, Q. Chen, Flow cytometry-based method facilitates optimization of PMA treatment condition for PMA-qPCR method. Molecular \& Cellular Probes (2018) S0890850818300756.

[8] K.M. Mckinnon, Flow Cytometry: An Overview. Other 120 (2018).

[9] M. Oravcova, M.N. Boddy, Recruitment, loading, and activation of the Smc5-Smc6 SUMO ligase. Curr Genet 65 (2019) 669-676.

[10] C.U. Martensson, C. Priesnitz, J. Song, L. Ellenrieder, K.N. Doan, F. Boos, A. Floerchinger, N. Zufall, S. Oeljeklaus, B. Warscheid, T. Becker, Mitochondrial protein translocation-associated degradation. Nature 569 (2019) 679-683.

[11] Y.T. Wang, W.Y. Hsiao, S.W. Wang, The fission yeast Pin1 peptidyl-prolyl isomerase promotes dissociation of Sty1 MAPK from RNA polymerase II and recruits Ssu72 phosphatase to facilitate oxidative stress induced transcription. Nucleic Acids Res (2021). 
[12] R.E. Workman, T. Pammi, B.T.K. Nguyen, L.W. Graeff, E. Smith, S.M. Sebald, M.J. Stoltzfus, C.W. Euler, J.W. Modell, A natural single-guide RNA repurposes Cas9 to autoregulate CRISPR-Cas expression. Cell (2021).

[13] X. Zhao, X. Li, P. Zhang, C. Li, W. Feng, X. Zhu, D. Wei, Effects of 5'-3' Exonuclease Xrn1 on Cell Size, Proliferation and Division, and mRNA Levels of Periodic Genes in Cryptococcus neoformans. Genes (Basel) 11 (2020).

[14] M.Q. Claire, G. Allen, C. Vasiliy, K. Lee, B.A. Cimini, K.W. Karhohs, D. Minh, L. Ding, S.M. Rafelski, T. Derek, CellProfiler 3.0: Next-generation image processing for biology. Plos Biology 16 (2018) e2005970.

[15] D. Houzet, S. Huet, A. Rahman, SysCellC: a data-flow programming model on multi-GPU. Procedia Computer Science 45 (2010).

[16] X. Li, H. Yang, H. Huang, T. Zhu, CELLCOUNTER: Novel open-source software for counting cell migration and invasion in vitro. Journal of Biomedicine and Biotechnology 2014 (2014) 863564.

[17] G. Quentin, M.R. Mh., OpenCFU, a New Free and Open-Source Software to Count Cell Colonies and Other Circular Objects. Plos One 8 (2013) e54072.

[18] I.V. Grishagin, Automatic cell counting with ImageJ. Analytical Biochemistry 473 (2015) 63-65.

[19] S. Berg, D. Kutra, T. Kroeger, C.N. Straehle, B.X. Kausler, C. Haubold, M. Schiegg, J. Ales, T. Beier, M. Rudy, ilastik: interactive machine learning for (bio)image analysis. Nature Methods (2019).

[20] L. Fiaschi, R. Nair, U. Koethe, F.A. Hamprecht, Learning to count with regression forest and structured labels, International Conference on Pattern Recognition, 2012.

Figures and Figure legends

Figure 1

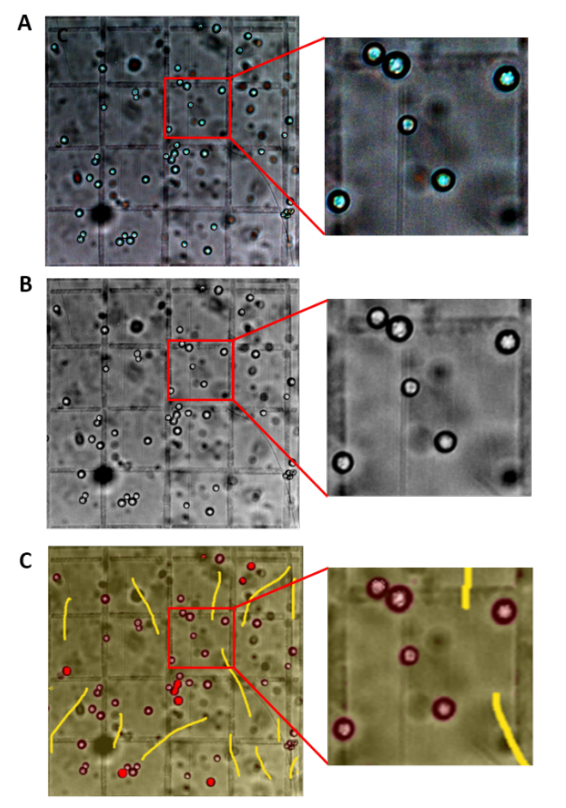

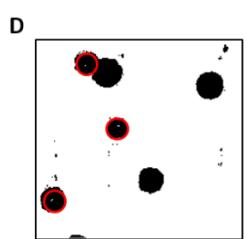

E
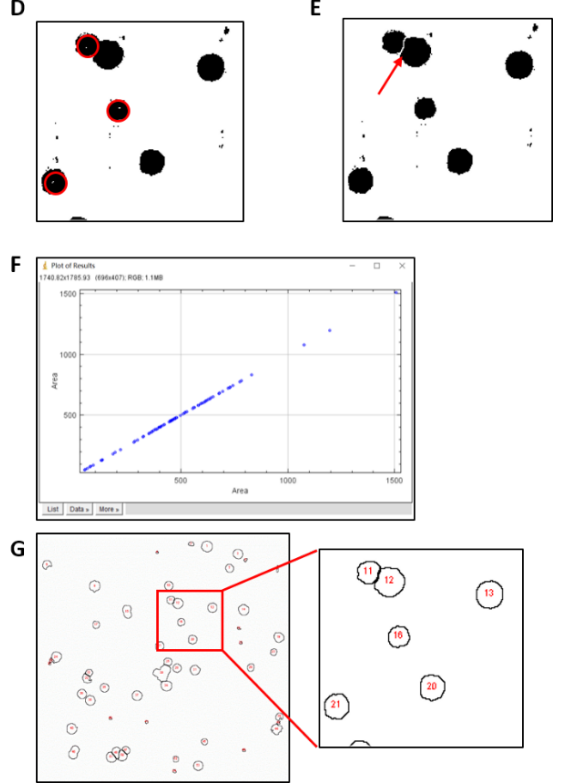

Figure1. Image capture and processing for the samples under complicated backgrounds.

Cells in suspensions were deposited into a hemocytometer, and images of complex background was taken and cropped to $1 \mathrm{x} 1 \mathrm{~mm}$. Representative images of one area with 16 smallest counting chambers are shown. 
Panel A shows the original RGB image captured by microscope. Panel B, the image was converted to 8bit and resized. After compression, machine learning-based ilastik was used to distinguish the background from yeast cells. Panel $\mathrm{C}$ shows the process that a user-defined class label was attached to the images with complex background. Whereafter, ImageJ macro was used to optimize the batch of images. Black-and-white images were presented first. Panel D shows the operation to fill the gap with the function of ImageJ, which are marked by the red circles. Panel E shows merging cells split by a single pixel line via the "Watershed" function, which are marked by red clipper. Area can be used to assess the objectives in images with ImageJ tool. The Area command was applied in panel F via the "Analyse Particles" function. After setting the threshold in the Analyse Particles command, cells counted automatically are highlighted and numbered in an overlay on the image as indicated in panel G.

\section{Figure 2}
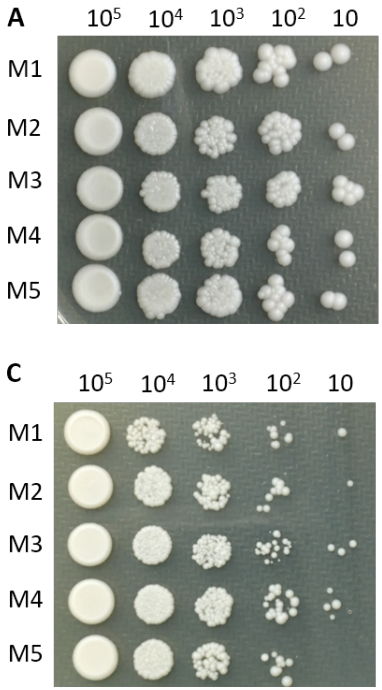
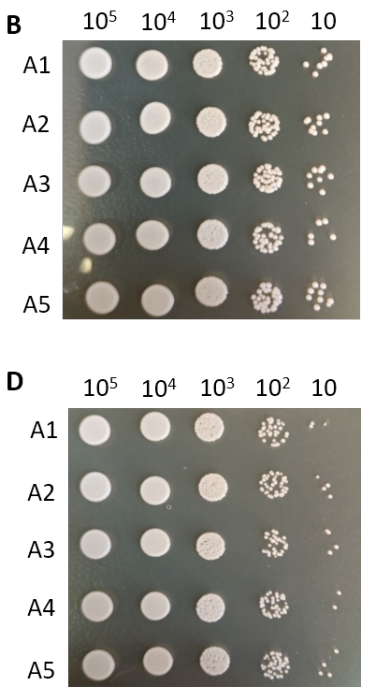

\section{Figure2. Spotting test}

5 out of 12 yeast samples in complex backgrounds or not were taken and diluted to $2 \times 10^{7}$ cells per ml, and then the diluent samples were subjected to 10 -fold dilutions. $5 \mu$ of each dilution were spotted onto YPD solid media. Thus, the numbers at the top of images indicate the CFUs in theory. (A) Manual counting results for samples of simple backgrounds. (B) Automatic counting results for samples of simple backgrounds with "Yeast Counter". (C) Manual counting results for samples of complex backgrounds. (D) Automatic counting results with ilastik and ImageJ for samples under complex backgrounds. 
Figure3

A

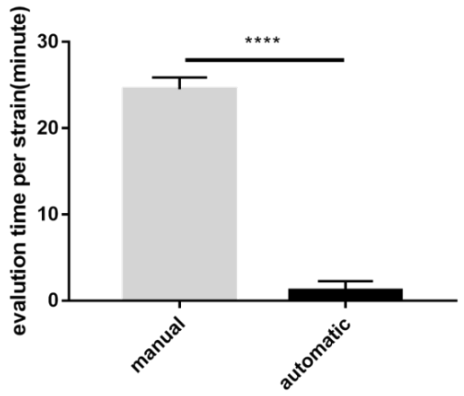

B

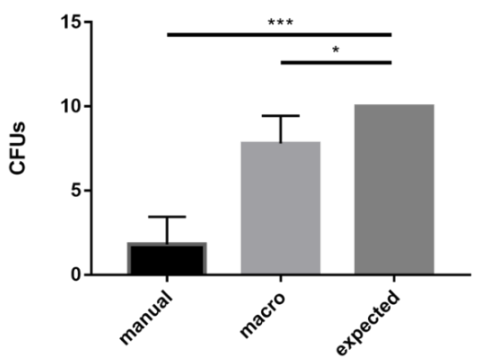

Figure 3. Comparation of time and accuracy between manual and automatic counting.

(A) The averaged evaluation times of both manual and automatized analysis ("yeast counter") of the same sample. Student's t-test (two-tailed), $\mathrm{p}<0.0001$. (B) The colony-forming units of the last spot in spotting test for both manual and automatic counting, and the comparation between experimental and theoretical value. The manual (left column) count is quite different from what is expected (right column), while automatic counting (middle column) is closer to the theoretical value. Continuous lines indicate statistically significant differences, asterisks indicate the according $\mathrm{p}$-value $(\mathrm{p}<0.001 ; \mathrm{p}<0.1$; Student's t-test (two-tailed). 